\title{
Retinopathy of Prematurity Requiring Treatment Is Closely Related to Head Growth during Neonatal Intensive Care Unit Hospitalization in Very Low Birth Weight Infants
}

\author{
Seong Phil Bae ${ }^{a}$ Ee-Kyung Kim ${ }^{b}$ Jungha Yun ${ }^{c}$ Young Mi Yoon ${ }^{d}$ \\ Seung Han Shin ${ }^{b}$ Su Yeon Park ${ }^{\mathrm{e}}$ \\ ${ }^{a}$ Department of Pediatrics, Soonchunhyang University College of Medicine, Soonchunhyang University Seoul \\ Hospital, Seoul, Republic of Korea; bepartment of Pediatrics, Seoul National University College of Medicine, Seoul \\ National University Children's Hospital, Seoul, Republic of Korea; 'Department of Pediatrics, CHA University School \\ of Medicine, CHA Ilsan Medical Center, Goyang-si, Republic of Korea; ${ }^{\mathrm{d} D e p a r t m e n t}$ of Pediatrics, Jeju University \\ School of Medicine, Jeju National University Hospital, Jeju-si, Republic of Korea; ${ }^{\text {DDepartment }}$ of Data Innovation, \\ Soonchunhyang University Seoul Hospital, Seoul, Republic of Korea
}

\section{Keywords}

Retinopathy of prematurity · Infant · Very low birth weight . Risk factors · Anthropometry

\begin{abstract}
Background: Retinopathy of prematurity (ROP) is caused by prenatal sensitization and postnatal insults to the immature retina. This process can be associated with the postnatal growth of preterm infants. We investigated whether ROP requiring treatment was associated with the postnatal growth failure of very low birth weight (VLBW) infants. Method: From a cohort of VLBW infants (birth weight $<1,500 \mathrm{~g}$ ) registered in the Korean Neonatal Network from January 2013 to December 2017, 3,133 infants with gestational age (GA) between 24 and 28 weeks were included in the study. Postnatal growth failure was defined when the change in each anthropometric $z$-score between birth and discharge was $<10$ th percentile of the total population. Propensity score matching (PSM) at 1:1 was performed to match the distribution of GA and postnatal morbidities between infants with and without ROP requiring treatment. Prenatal factors and ROP
\end{abstract}

karger@karger.com www.karger.com/neo

Karger"

BOPEN ACCESS
(C) 2022 The Author(s)

Published by S. Karger AG, Basel

This is an Open Access article licensed under the Creative Commons Attribution-NonCommercial-4.0 International License (CC BY-NC) (http://www.karger.com/Services/OpenAccessLicense), applicable to the online version of the article only. Usage and distribution for commercial purposes requires written permission. were analyzed by conditional logistic regression. Results: Of 3,133 enrolled infants, 624 (19.9\%) were diagnosed with ROP requiring treatment. After PSM, ROP requiring treatment was associated with postnatal growth failure assessed by head circumference (adjusted odds ratio [aOR] 1.91, 95\% confidence interval [Cl] 1.18-3.09), but not weight (aOR 1.45, $95 \% \mathrm{Cl} 0.97-2.17$ ) and length (aOR 1.21, 95\% Cl 0.81-1.82). Conclusion: ROP requiring treatment was associated with poor head circumference growth, not with weight and length. Our findings suggest that ROP requiring treatment and poor head growth during NICU hospitalization are fundamentally related.

(c) 2022 The Author(s)

Published by S. Karger AG, Basel

\section{Introduction}

Retinopathy of prematurity (ROP) is a complication of prematurity caused by prenatal sensitization and postnatal insults to the immature retina that not only leads to visual disabilities but also is reportedly associated with poor neurodevelopmental outcomes [1,2]. Small for ges-
Correspondence to:

Ee-Kyung Kim, kimek@snu.ac.kr 
Fig. 1. Flowchart of the study population. BPD, bronchopulmonary dysplasia; NEC, necrotizing enterocolitis; PDA, patent ductus arteriosus; PMA, postmenstrual age; ROP, retinopathy of prematurity; VLBW, very low birth weight.
9,374 preterm VLBW infants

Did not meet the gestational age criteria $(n=4,547)$

Died before 36 weeks PMA $(n=945)$

Congenital anomalies ( $n=82$ )

Transferred to other hospitals $(n=170)$

Incomplete anthropometric data $(n=492)$

Unknown status for ROP requiring treatment $(n=5)$

3,133 infants enrolled

1:1 propensity score matching for gestational age and postnatal morbidities;

postnatal corticosteroids for BPD, surgically treated PDA,

moderate-to-severe BPD, severe brain injury, sepsis and NEC

Infants requiring ROP treatment

$(n=472)$

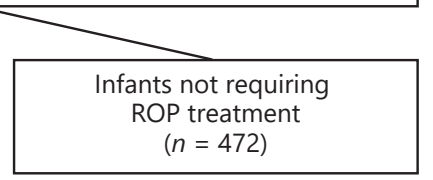

tational age, along with low gestational age (GA), is the most commonly reported significant risk factor for ROP [3]. In addition, low serum levels of insulin-like growth factor 1 (IGF-1) are known to be related to the development of ROP, and many studies are developing efficient ROP screening criteria by modeling to predict the occurrence of severe ROP using GA and postnatal weight gain instead of measuring IGF-1 levels [4-6]. However, few studies have investigated the relationship between ROP requiring treatment and postnatal growth in various anthropometric modes during NICU hospitalization, controlling for major comorbidities affecting growth.

Preterm infants with ROP requiring treatment are often preceded or accompanied by comorbidities such as intraventricular hemorrhage (IVH), sepsis, necrotizing enterocolitis (NEC), and bronchopulmonary dysplasia (BPD). These illnesses frequently hamper optimal nutritional support and are known to affect growth by lowering IGF-1 levels [7]. These morbidities are also related to postnatal inflammation and have been shown to be associated with severe ROP [8-10]. Studying the relationship between ROP requiring treatment and various postnatal anthropometric changes after controlling for the major prematurity-associated morbidities is important because it can help to determine which aspect of growth is associated with ROP.

Since 2013, the Korean Neonatal Network (KNN) has collected data from 70 hospitals to investigate the factors related to mortality and morbidity in Korean VLBW infants [11]. We investigated the association between ROP requiring treatment and changes in postnatal anthropometric values in KNN data with 1:1 propensity score matching (PSM) to match the distribution of GA and postnatal morbidities in infants born between 24 and 28 weeks of GA.

\section{Materials and Methods}

\section{Study Population}

VLBW infants (birth weight $<1,500 \mathrm{~g}$ ) born in or transferred to the participating NICU of the KNN within 28 days after birth between January 2013 and December 2017 were prospectively registered in the KNN database by the staff at each hospital using a standardized electronic case report form. Infants whose parents did not want to register in the KNN were excluded. The inclusion criteria of this study were infants with GAs between 24 and 28 weeks. Infants with major congenital anomalies or who died before the postmenstrual age (PMA) of 36 weeks were excluded.

Of the total group of 9,374 VLBW infants, 4,547 who did not meet the GA criteria, 945 who died before 36 weeks PMA, 82 who had major congenital anomalies, 497 with incomplete data missing anthropometric values or ROP therapy, and 170 whose ROP status was not obtainable due to transfer to other hospitals after 28 days of birth were excluded. Finally, 3,133 VLBW infants were analyzed (shown in Fig. 1). The KNN registry was approved by the Institutional Review Board (IRB), and informed consent was obtained from parents upon enrollment at each participating hospital. 


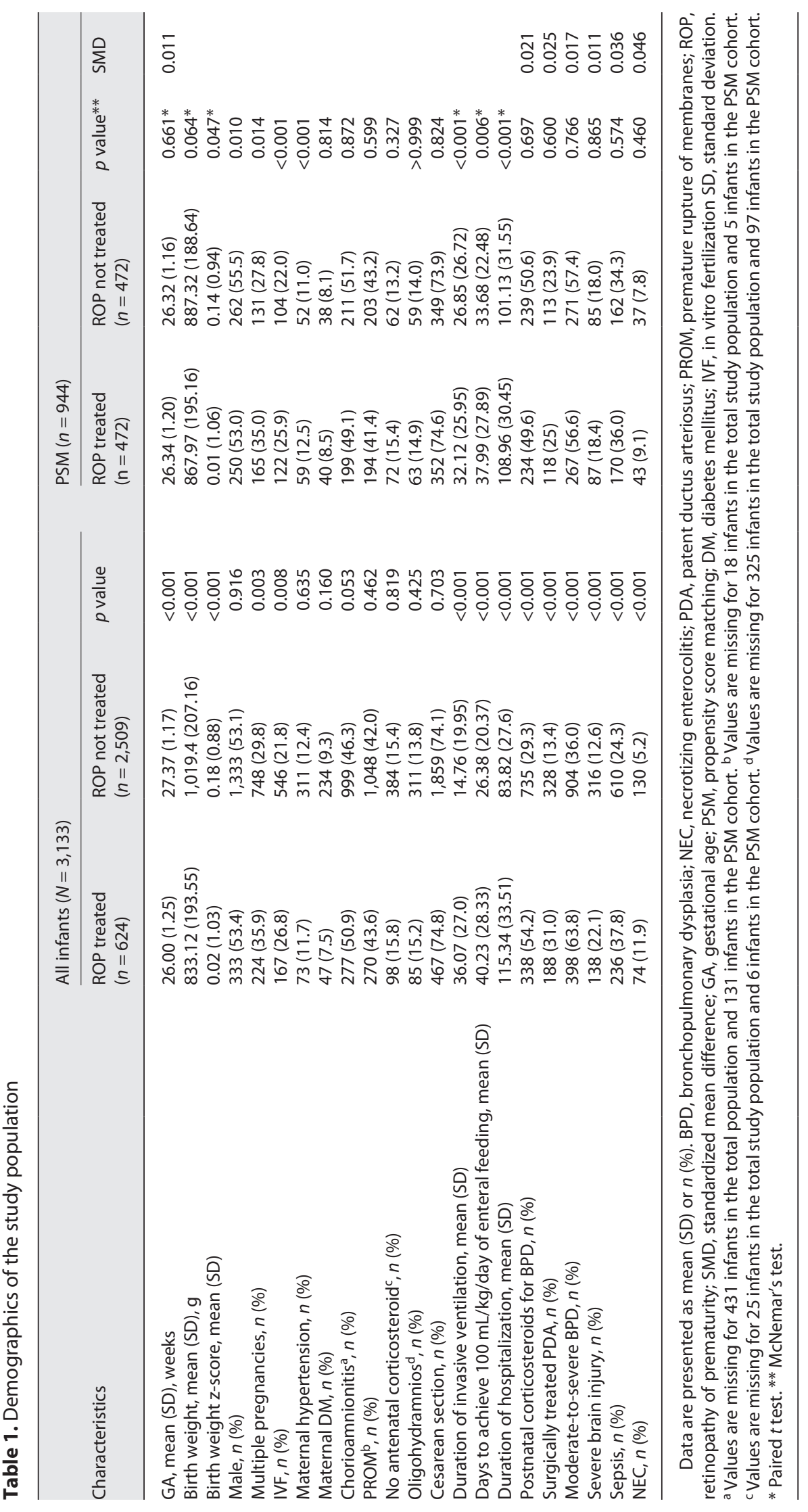




\section{Definition}

We used only the z-scores of the anthropometric values for GA based on the INTERGROWTH-21st standard, instead of percentile or raw data, at birth and discharge [12]. The $\mathrm{z}$-scores of weight, length, and head circumference at birth were subtracted from those $\mathrm{z}$-scores at discharge. Postnatal growth failure was defined when the change in each anthropometric $\mathrm{z}$-score between birth and discharge was $<10$ th percentile of 3,133 infants enrolled in the study.

GA was determined either through obstetric examination by ultrasound at early pregnancy or based on the obstetric record of the last menstrual period of the mother. Maternal hypertension included hypertension before pregnancy and pregnancy-induced hypertension. Maternal diabetes mellitus (DM) included DM and gestational DM. Chorioamnionitis was based on histologic findings of the presence of acute inflammatory changes in the choriondecidua, amnion, umbilical cord, and chorionic plate by pathologists at each participating facility based on the criteria of Salafia et al. modified by Yoon et al. $[13,14]$. BPD was defined as moderateto-severe BPD using the criteria from the National Institute of Child Health Workshop [15]. Surgically treated patent ductus arteriosus (PDA) was defined as symptomatic PDA requiring surgical treatment. Severe brain injury included grades 3-4 IVH according to the Papile classification, post-hemorrhagic hydrocephalus, and cystic periventricular leukomalacia using brain sonography or brain magnetic resonance imaging [16]. NEC was defined as stage 2 or higher according to Bell's criteria [17]. ROP treatment was determined by ophthalmologists at each participating facility using Early Treatment for Retinopathy of Prematurity criteria [18]. ROP requiring treatment was defined as an operation (cryotherapy, laser photocoagulation, or vitrectomy) or intravitreal injection with anti-vascular endothelial growth factor (antiVEGF) [19].

\section{Statistical Analysis}

To compare the characteristics of infants with and without ROP requiring treatment in the total population, the continuous variables were analyzed using $t$ tests or the Mann-Whitney test and expressed as means and standard deviation (SD). The categorical variables were analyzed by $\chi^{2}$ tests and expressed as numbers and percentile. To analyze the relationship between each anthropometric value for postnatal growth failure and ROP requiring treatment, 1:1 PSM was performed including GA and the outcomes of postnatal corticosteroids administration for BPD, surgically treated PDA, moderate-to-severe BPD, severe brain injury, sepsis, and NEC using a nearest neighbor approach with a caliper restriction to minimize the effect of other variables confounding the relationship of $\mathrm{ROP}$ requiring treatment and growth. To compare the demographics of the groups after PSM, the paired $t$ test and McNemar's test were used. Univariable conditional logistic regression analysis was performed separately to identify the risk factors that affected each postnatal growth failure. Multivariable conditional logistic regression was used to adjust for variables (male gender, z-score at birth, in vitro fertilization [IVF], maternal hypertension, premature rupture of membranes [PROM], the duration of invasive ventilation, days to achieve $100 \mathrm{~mL} / \mathrm{kg} /$ day of enteral feeding, and the duration of hospitalization) that showed statistically significant differences with a $p$ value of $<0.1$ in univariable analysis. A $p$ value of $<0.05$ was considered statistically significant. Statistical analyses were performed by Rex (Version 3.0.3; RexSoft Inc., Seoul, Korea).

ROP Requiring Treatment Associated with Poor Head Growth

\section{Results}

A total of 3,133 VLBW infants fulfilled the inclusion criteria, and 624 (19.9\%) were diagnosed with ROP requiring treatment. Among 624 infants, 101 (16.2\%) received both laser photocoagulation and anti-VEGF therapy, 368 (59.0\%) received only laser photocoagulation, and $113(18.1 \%)$ received only anti-VEGF therapy. The remaining 42 infants did not have procedural data.

The characteristics of the VLBW infants enrolled in this study indicated a significant difference in neonatal characteristics and comorbidities between infants requiring and not requiring ROP treatment. Infants requiring ROP treatment had lower GA and birth weight z-scores than infants not requiring ROP treatment. The rate of multiple pregnancies, IVF, the duration of invasive ventilation, days to achieve $100 \mathrm{~mL} / \mathrm{kg} /$ day of enteral feeding, the duration of hospitalization, postnatal corticosteroids administration for BPD, surgically treated PDA, moderate-to-severe BPD, severe brain injury, sepsis, and NEC were higher in infants requiring ROP treatment than in infants not requiring ROP treatment (Table 1).

In the total study population, postnatal growth was greatly suppressed in the order of length (mean $-1.68 \mathrm{SD}$ $1.7)$, head circumference $(-1.28, \mathrm{SD} 1.6)$, and weight $(-1.05$, SD 1.1) ( $p<0.001$, data not shown). All anthropometric values were lower in infants requiring ROP treatment at birth and discharge than in infants not requiring ROP treatment (Table 2). In the propensity score-matched cohort, the order of suppression of postnatal growth was the same, but the degree of suppression was greater than that of the total study population (length, mean -2.06 , SD 1.8; head circumference, -1.58 , SD 1.8; weight, -1.29 , SD 1.2; $p$ $<0.001$, data not shown). All anthropometric values except length at birth were significantly lower in infants requiring ROP treatment than those not requiring ROP treatment.

In the univariable conditional analysis of the factors not involved in PSM, $\mathrm{z}$-score at birth, the duration of invasive ventilation, the duration of hospitalization, and ROP requiring treatment were shown to be risk factors for postnatal growth failure assessed by weight. In postnatal growth failure assessed by length, IVF, maternal hypertension, the duration of invasive ventilation, days to achieve $100 \mathrm{~mL} /$ $\mathrm{kg} /$ day of enteral feeding, and the duration of hospitalization were significant risk factors. Male gender, z-score at birth, the duration of invasive ventilation, days to achieve $100 \mathrm{~mL} / \mathrm{kg} /$ day of enteral feeding, the duration of hospitalization, and ROP requiring treatment were associated with postnatal growth failure assessed by head circumference (Table 3). Multivariable conditional logistic regression 
Table 2. Trend of z-score changes in each anthropometric marker over time

\begin{tabular}{|c|c|c|c|c|c|c|}
\hline \multirow[b]{2}{*}{ z-score } & \multicolumn{3}{|c|}{ Study population $(N=3,133)$} & \multicolumn{3}{|l|}{$\operatorname{PSM}(n=944)$} \\
\hline & $\begin{array}{l}\text { ROP treated } \\
(n=624)\end{array}$ & $\begin{array}{l}\text { ROP not treated } \\
(n=2,509)\end{array}$ & $p$ value $^{*}$ & $\begin{array}{l}\text { ROP treated } \\
(n=472)\end{array}$ & $\begin{array}{l}\text { ROP not treated } \\
(n=472)\end{array}$ & $p$ value $* *$ \\
\hline \multicolumn{7}{|l|}{ At birth } \\
\hline Weight & $0.02(1.03)$ & $0.18(0.88)$ & $<0.001$ & $0.01(1.06)$ & $0.14(0.94)$ & 0.047 \\
\hline Length & $-0.45(0.92)$ & $-0.23(0.89)$ & $<0.001$ & $-0.43(0.95)$ & $-0.33(0.88)$ & 0.114 \\
\hline Weight & $-1.50(1.37)$ & $-0.75(1.23)$ & $<0.001$ & $-1.45(1.37)$ & $-0.99(1.33)$ & $<0.001$ \\
\hline Length & $-2.79(2.01)$ & $-1.75(1.82)$ & $<0.001$ & $-2.69(2.01)$ & $-2.19(1.97)$ & $<0.001$ \\
\hline $\mathrm{HC}$ & $-2.21(1.91)$ & $-1.24(1.52)$ & $<0.001$ & $-2.09(1.97)$ & $-1.57(1.70)$ & $<0.001$ \\
\hline \multicolumn{7}{|c|}{ Postnatal growth ${ }^{a}$} \\
\hline Weight & $-1.52(1.22)$ & $-0.93(1.02)$ & $<0.001$ & $-1.46(1.18)$ & $-1.13(1.17)$ & $<0.001$ \\
\hline Length & $-2.34(1.74)$ & $-1.52(1.60)$ & $<0.001$ & $-2.26(1.72)$ & $-1.86(1.74)$ & $<0.001$ \\
\hline
\end{tabular}

Data are presented as mean (SD). HC, head circumference; ROP, retinopathy of prematurity; PSM, propensity score matching; SD, standard deviation. ${ }^{a}$ Change in $z$-scores from birth to discharge. ${ }^{*}$ Student's $t$ test. ${ }^{*}$ Paired $t$ test.

Table 3. Univariable conditional logistic regression for postnatal growth failure in propensity score-matched cohort $(n=944)$

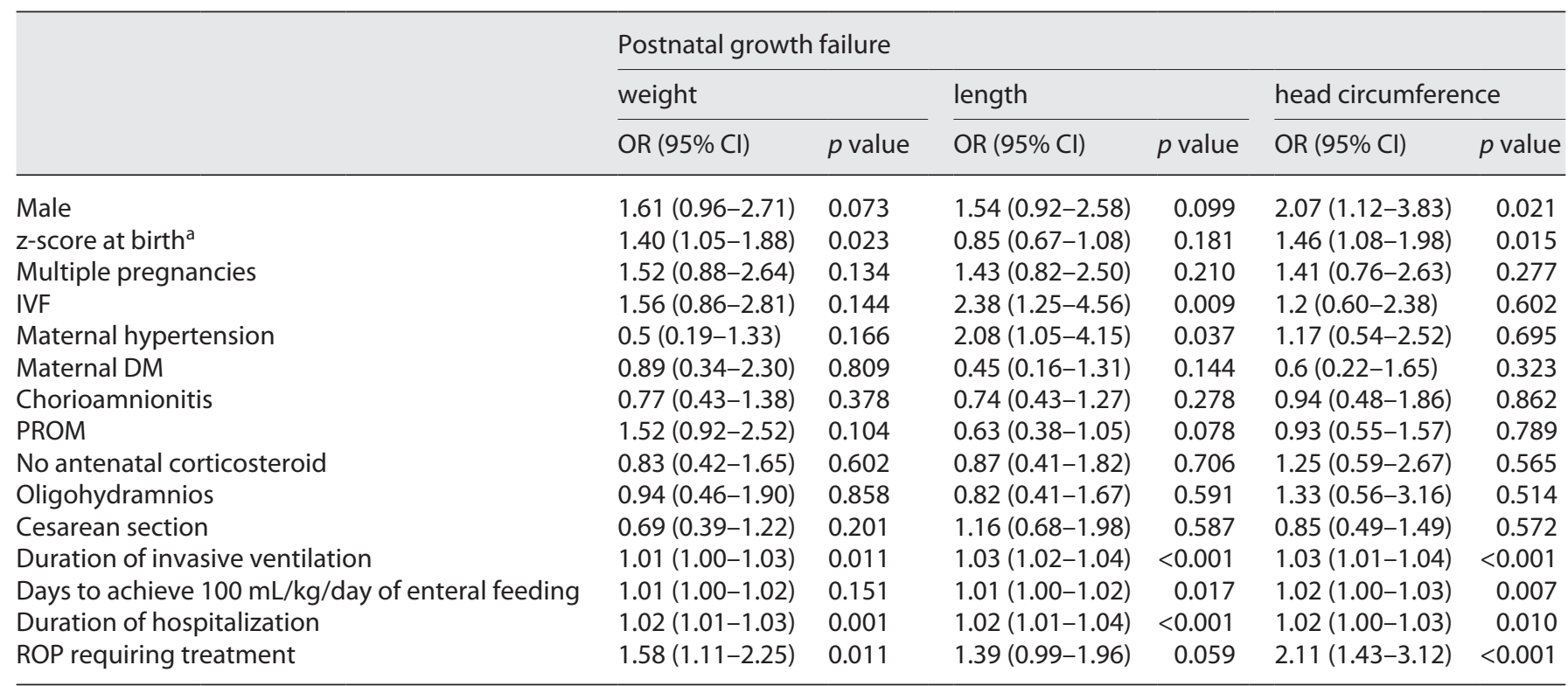

$\mathrm{Cl}$, confidence interval; OR, odds ratio; PROM, premature rupture of membranes; DM, diabetes mellitus; IVF, in vitro fertilization. ${ }^{\mathrm{a}} \mathrm{Z}$-score at birth in each anthropometric mode.

analysis adjusted for the confounding variables of postnatal growth failure revealed that ROP requiring treatment was associated with postnatal growth failure assessed by head circumference (adjusted odds ratio [aOR] 1.91, 95\% confidence interval [CI] 1.18-3.09, $p=0.008$ ), but not weight (aOR 1.45, 95\% CI 0.97-2.17, $p=0.070$ ) or length (aOR 1.21, 95\% CI 0.81-1.82, $p=0.350$; Table 4 ).

\section{Discussion}

This study demonstrated that ROP requiring treatment was associated with postnatal growth failure assessed by head circumference, but not by weight and length, when GA, prematurity-related morbidities, and prenatal factors were controlled in the propensity score- 
Table 4. Multivariable conditional logistic regression analysis of the association between ROP requiring treatment and postnatal growth failure

\begin{tabular}{|c|c|c|c|c|c|c|}
\hline & \multicolumn{6}{|c|}{ Postnatal growth failure } \\
\hline & \multicolumn{2}{|l|}{ weight } & \multicolumn{2}{|l|}{ length } & \multicolumn{2}{|c|}{ head circumference } \\
\hline & aOR $(95 \% \mathrm{Cl})$ & $p$ value $e^{*}$ & aOR $(95 \% \mathrm{Cl})$ & $p$ value* & aOR $(95 \% \mathrm{Cl})$ & $p$ value* \\
\hline ROP requiring treatment & $1.45(0.97-2.17)$ & 0.070 & $1.21(0.81-1.82)$ & 0.350 & $1.91(1.18-3.09)$ & 0.008 \\
\hline
\end{tabular}

aOR, adjusted odds ratio; $\mathrm{Cl}$, confidence interval; ROP, retinopathy of prematurity; IVF, in vitro fertilization. ${ }^{*}$ Adjusted for male gender, $z$-score at birth, IVF, maternal hypertension, premature rupture of membranes, the duration of invasive ventilation, days to achieve 100 $\mathrm{mL} / \mathrm{kg} /$ day of enteral feeding, and the duration of hospitalization.

matched cohort. The disproportionate growth patterns of preterm infants such as more weight gain relative to length gain [20] or suboptimal head growth [21] during NICU hospitalization, especially in a more immature population [22], were constantly observed even though advanced nutritional support was provided. Thus, interest in the effect of non-nutritional factors such as inflammation and infection on growth is increasing.

Among the risk factors for ROP, prenatal inflammation and infection are known to sensitize the retina to subsequent insults rather than directly triggering ROP [9]. In addition, a study recently reported that postnatal factors related to inflammation such as sepsis, NEC, grades 3-4 IVH, and supplemental oxygen on day 28 were more strongly correlated with severe ROP than prenatal inflammation-related factors [23]. Those morbidities may also adversely affect nutritional support by impaired lipid tolerance, the withholding of enteral feeding, and volume restriction, and can lead to poor growth of prematurity by disrupting metabolic-endocrine homeostasis under these postnatal stress and undernutrition conditions [24]. We conducted PSM to minimize the effect of these comorbidities known to be related to postnatal growth failure to identify the relationship between ROP requiring treatment and postnatal growth.

In this study, the average PMA at discharge was $41.3 \pm$ 4.14 weeks (mean $\pm \mathrm{SD}$ ). In both the total study population and the propensity score-matched cohort, infants with ROP requiring treatment had significantly lower birth weight and postnatal weight growth than infants not requiring ROP treatment. This is consistent with previous reports that appointed postnatal weight gain as one of the criteria for efficient ROP screening [4-6] and a large cohort study [25]. However, those studies were not intended to search for the independent relationship between postnatal weight gain and ROP and did not control for poten-

ROP Requiring Treatment Associated with Poor Head Growth tial confounders, especially comorbidities. Our multivariate analysis showed that $\mathrm{ROP}$ requiring treatment was not associated with postnatal growth failure assessed by weight or length but with head circumference. Li et al. [26] also showed that low weight velocity from day 7 to day 28 was associated with ROP, but in a multivariable logistic regression analysis adjusting for GA, BPD, and surgical ligation for PDA, it was no longer significant. The results of this study indicated a close relationship between ROP requiring treatment and poor head growth, independent of the comorbidities, suggesting a shared pathogenetic progression between ROP and head growth failure.

Löfqvist et al. [27] reported that head growth decelerated after birth until 30-32 weeks of PMA, and the degree of head growth retardation during those periods corresponded to the severity of ROP and the suppression of serum IGF-1 levels. They suggested that retinal vascular growth retardation that initiates ROP is paralleled by brain growth deceleration. In this study, we could not analyze head circumference growth divided by periods. Nevertheless, the study results showed that the severity of ROP was correlated with head circumference growth during the entire hospitalization period. The results of this national cohort study are consistent with previous studies on the relationship between ROP and head growth $[27,28]$ by showing that ROP requiring treatment was associated with postnatal head growth failure during NICU hospitalization. This is also consistent with reports that ROP was associated with poor neurodevelopmental outcomes $[1,2,29]$ since reduced head growth before term was associated with later suboptimal neurodevelopment in preterm infants $[30,31]$.

There are several limitations of this study. First, $\mathrm{z}$ scores were measured using the INTERGROWTH-21st standard, which had not previously been validated in Korean preterm infants. However, in a recent study using 
KNN data, the association of extrauterine growth restriction defined by INTERGROWTH-21 st growth chart with neonatal morbidity was well demonstrated [32]. There was a significant difference in the magnitude of $\mathrm{z}$-score changes between the INTERGROWTH-21st standard and the Fenton chart [32], therefore, we defined postnatal growth failure as a change in $\mathrm{z}$-score of $<10$ th percentile of the 3,133 infants enrolled in the study. Thus, caution is needed when interpreting these results.

Second, since only anthropometric values at birth and discharge were collected in the KNN registry, it was impossible to measure changes in postnatal growth failure within some specific period of PMA. Third, the collection of information on the date of treatment, zone, and plus disease began in 2015 in the $\mathrm{KNN}$, and $65 \%$ of the infants in this study lacked these data, which precluded more detailed analyses. Last, postnatal growth failure is known to be affected by nutritional and non-nutritional factors. However, detailed nutritional information was not available in the network database.

\section{Conclusion}

ROP requiring treatment was associated with poor head circumference growth, not with weight and length. Our findings suggest that ROP requiring treatment and poor head growth during NICU hospitalization are fundamentally related.

\section{Acknowledgment}

This research was supported by a fund (2019-ER7103-02\#) by Research of Korea National Institute of Health.

\section{Statement of Ethics}

The study conformed to the ethical guidelines of the World Medical Association Declaration of Helsinki. The registration of data in the KNN was approved by the IRB of each participating center. Informed consent was obtained from the parents of each infant prior to participation in the KNN registry. This study was approved by the IRB of Seoul National University Hospital (IRB No. 1303-092-476).

\section{Conflict of Interest Statement}

The authors have no conflicts of interest to declare.

\section{Funding Sources}

This research was supported by Soonchunhyang University Research Fund to cover publication cost and Basic Science Research Program through the National Research Foundation of Korea funded by the Ministry of Education (NRF-2017R1D1A1B04030931) to cover English proofreading cost. The funders had no role in the study design, data collection and analysis, decision to publish, or preparation of the manuscript.

\section{Author Contributions}

S.P.B. and E.K.K. conceptualized and designed the research. S.P.B., Y.M.Y., and S.Y.P. collected and analyzed the data. S.P.B. and J.H.Y. wrote the manuscript. S.H.S assisted with study design and reviewed the manuscript. E.K.K. reviewed and revised the manuscript. All authors read and approved the final manuscript.

\section{Data Availability Statement}

The KNN Publication Ethics Policy adheres to the following research data management and access guidelines: all information about patients and participating NICUs is confidential and may be used by individuals for approved research purposes. If an individual or institution that is not affiliated with KNN wants to use the data, it must make an official request to the KNN Publication Ethics Committee and obtain approval from the network.

\section{References}

1 Glass TJA, Chau V, Gardiner J, Foong J, Vinall J, Zwicker JG, et al. Severe retinopathy of prematurity predicts delayed white matter maturation and poorer neurodevelopment. Arch Dis Child Fetal Neonatal Ed. 2017 Nov; 102(6):F532-F7.

2 Drost FJ, Keunen K, Moeskops P, Claessens NHP, van Kalken F, Išgum I, et al. Severe retinopathy of prematurity is associated with reduced cerebellar and brainstem volumes at term and neurodevelopmental deficits at 2 years. Pediatr Res. 2018 Apr 01;83(4):818-24.

3 Boghossian NS, Geraci M, Edwards EM, Horbar JD. Morbidity and mortality in small for gestational age infants at 22 to 29 weeks' gestation. Pediatrics. 2018;141(2):e20172533.

4 Biniwale M, Weiner A, Sardesai S, Cayabyab R, Barton L, Ramanathan R. Early postnatal weight gain as a predictor for the development of retinopathy of prematurity. J Matern Fetal Neonatal Med. 2019 Feb;32(3):429-33.

5 Lin L, Binenbaum G. Postnatal weight gain and retinopathy of prematurity. Semin Perinatol. 2019 Oct;43(6):352-9.

6 Binenbaum G, Tomlinson LA, de Alba Campomanes AG, Bell EF, Donohue P, Morrison $D$, et al. Validation of the postnatal growth and retinopathy of prematurity screening cri- teria. JAMA Ophthalmol. 2020 Jan 1;138(1): 31-7.

7 Hellström A, Engström E, Hård AL, Albertsson-Wikland K, Carlsson B, Niklasson A, et al. Postnatal serum insulin-like growth factor I deficiency is associated with retinopathy of prematurity and other complications of premature birth. Pediatrics. 2003 Nov;112(5): 1016-20.

8 Leviton A, Dammann O, Engelke S, Allred E, Kuban KC, O'Shea TM, et al. The clustering of disorders in infants born before the 28th week of gestation. Acta Paediatr. 2010 Dec; 99(12):1795-800. 
9 Lee J, Dammann O. Perinatal infection, inflammation, and retinopathy of prematurity. Semin Fetal Neonatal Med. 2012 Feb;17(1): 26-9.

10 Zozaya C, Avila-Alvarez A, Arruza L, GarcíaMuñoz Rodrigo F, Fernandez-Perez C, Castro A, et al. The Effect of morbidity and sex on postnatal growth of very preterm infants: a Multicenter Cohort Study. Neonatology. 2019;115(4):348-54.

11 Chang YS, Park HY, Park WS. The Korean neonatal network: an overview. J Korean Med Sci. 2015;30(Suppl 1):S3-S11.

12 Villar J, Cheikh Ismail L, Victora CG, Ohuma EO, Bertino E, Altman DG, et al. International standards for newborn weight, length, and head circumference by gestational age and sex: the Newborn Cross-Sectional Study of the INTERGROWTH-21st Project. Lancet. 2014 Sep 6;384(9946):857-68.

13 Salafia CM, Weigl C, Silberman L. The prevalence and distribution of acute placental inflammation in uncomplicated term pregnancies. Obstet Gynecol. 1989 Mar;73(3 Pt 1): 383-9.

14 Yoon BH, Romero R, Kim CJ, Jun JK, Gomez $\mathrm{R}, \mathrm{Choi} \mathrm{JH}$, et al. Amniotic fluid interleukin-6: a sensitive test for antenatal diagnosis of acute inflammatory lesions of preterm placenta and prediction of perinatal morbidity. Am J Obstet Gynecol. 1995 Mar;172(3):960-70.

15 Ehrenkranz RA, Walsh MC, Vohr BR, Jobe AH, Wright LL, Fanaroff AA, et al. Validation of the National Institutes of Health consensus definition of bronchopulmonary dysplasia. Pediatrics. 2005 Dec;116(6):1353-60.

16 Papile LA, Burstein J, Burstein R, Koffler H. Incidence and evolution of subependymal and intraventricular hemorrhage: a study of infants with birth weights less than 1,500 gm. J Pediatr. 1978 Apr;92(4):529-34.
17 Bell MJ, Ternberg JL, Feigin RD, Keating JP, Marshall R, Barton L, et al. Neonatal necrotizing enterocolitis. Therapeutic decisions based upon clinical staging. Ann Surg. 1978 Jan; 187(1):1-7.

18 Early Treatment For Retinopathy Of Prematurity Cooperative Group. Revised indications for the treatment of retinopathy of prematurity: results of the early treatment for retinopathy of prematurity randomized trial. Arch Ophthalmol. 2003;121(12):1684-94.

19 International Committee for the Classification of Retinopathy of Prematurity. The international classification of retinopathy of prematurity revisited. Arch Ophthalmol. 2005 Jul;123(7):991-9.

20 Fenton TR, Cormack B, Goldberg D, Nasser R, Alshaikh B, Eliasziw M, et al. "Extrauterine growth restriction" and "postnatal growth failure" are misnomers for preterm infants. J Perinatol. 2020 May 01;40(5):704-14.

21 Bouyssi-Kobar M, du Plessis AJ, McCarter R, Brossard-Racine M, Murnick J, Tinkleman L, et al. Third trimester brain growth in preterm infants compared with in utero healthy fetuses. Pediatrics. 2016 Nov; 138(5).

22 Franz AR, Pohlandt F, Bode H, Mihatsch WA, Sander S, Kron M, et al. Intrauterine, early neonatal, and postdischarge growth and neurodevelopmental outcome at 5.4 years in extremely preterm infants after intensive neonatal nutritional support. Pediatrics. 2009 Jan;123(1):e101-9.

23 Goldstein GP, Leonard SA, Kan P, Koo EB, Lee HC, Carmichael SL. Prenatal and postnatal inflammation-related risk factors for retinopathy of prematurity. J Perinatol. 2019 2019 Jul 01;39(7):964-73.

24 Möllers LS, Yousuf EI, Hamatschek C, Morrison KM, Hermanussen M, Fusch $\mathrm{C}$, et al. Metabolic-endocrine disruption due to preterm birth impacts growth, body composition, and neonatal outcome. Pediatr Res. 2021.
25 Klevebro S, Lundgren P, Hammar U, Smith LE, Bottai M, Domellöf M, et al. Cohort study of growth patterns by gestational age in preterm infants developing morbidity. BMJ Open. 2016;6(11):e012872.

26 Li Y, Shah M, Miller MR, Lee DSC, Sharan S. Impact of early postnatal weight gain on retinopathy of prematurity in very preterm infants in southwestern ontario. J Pediatr Ophthalmol Strabismus. 2019 May 22;56(3):168-72.

27 Löfqvist C, Engström E, Sigurdsson J, Hård AL, Niklasson A, Ewald U, et al. Postnatal head growth deficit among premature infants parallels retinopathy of prematurity and insulin-like growth factor-1 deficit. Pediatrics. 2006 Jun;117(6):1930-8.

28 Sveinsdóttir K, Ley D, Hövel H, Fellman V, Hüppi PS, Smith LEH, et al. Relation of retinopathy of prematurity to brain volumes at term equivalent age and developmental outcome at 2 years of corrected age in very preterm infants. Neonatology. 2018;114(1):46-52.

29 Schmidt B, Davis PG, Asztalos EV, Solimano A, Roberts RS. Association between severe retinopathy of prematurity and nonvisual disabilities at age 5 years. JAMA. 2014;311(5): 523-5.

30 Belfort MB, Rifas-Shiman SL, Sullivan T, Collins CT, McPhee AJ, Ryan P, et al. Infant growth before and after term: effects on neurodevelopment in preterm infants. Pediatrics. 2011 Oct;128(4):e899-906.

31 Sammallahti S, Pyhälä R, Lahti M, Lahti J, Pesonen $\mathrm{AK}$, Heinonen $\mathrm{K}$, et al. Infant growth after preterm birth and neurocognitive abilities in young adulthood. J Pediatr. 2014 Dec; 165(6):1109-e3.

32 Kim YJ, Shin SH, Cho H, Shin SH, Kim SH, Song IG, et al. Extrauterine growth restriction in extremely preterm infants based on the Intergrowth-21st Project Preterm Postnatal Follow-up Study growth charts and the Fenton growth charts. Eur J Pediatr. 2021 Mar; 180(3):817-24. 